\title{
Editorial: special issue on hot subdwarf stars
}

\author{
Zhanwen Han • Simon Jeffery • Philipp Podsiadlowski • \\ Michael A. Dopita
}

Received: 13 August 2010 / Accepted: 13 August 2010 / Published online: 14 September 2010

(C) Springer Science+Business Media B.V. 2010

We are pleased to present in this Special Issue of Astrophysics \& Space Science a compilation of the latest research on the fascinating subject of Hot subluminous stars (or hot subdwarfs). These stars are located between the upper main sequence and the white-dwarf sequence in the HertzsprungRussell diagram. As galactic field stars, they include the subdwarf $\mathrm{O}$ and $\mathrm{B}$ stars. However, they are also found in globular clusters where they are known as extreme horizontal branch (EHB) stars. The majority of these stars are believed to be helium-core-burning stars with extremely thin hydrogen envelopes $\left(<0.02 M_{\odot}\right)$. They play an important role in many aspects of astrophysics, helping to shed light on, for example the source of the UV continuum in Elliptical Galaxies, binary evolution, stellar structure, and the physical

\section{Z. Han $(\bowtie)$}

National Astronomical Observatories/Yunnan Observatory, Chinese Academy of Sciences, Kunming, 650011, China

e-mail: zhanwenhan@ynao.ac.cn

Z. Han

Key Laboratory for the Structure and Evolution of Celestial

Objects, Chinese Academy of Sciences, Kunming, 650011, China

S. Jeffery

Armagh Observatory, College Hill, Armagh BT61 9DG,

$\mathrm{N}$. Ireland

e-mail: csj@arm.ac.uk

P. Podsiadlowski

Sub-department of Astrophysics, Oxford University, Keble Road, Oxford, OX1 3RH, UK

e-mail: podsi@astro.ox.ac.uk

M.A. Dopita

Mt. Stromlo Observatory, RSAA,

The Australian National University, Cotter Road, Weston Creek, ACT 2611, Australia

e-mail: michael.dopita@anu.edu.au processes operating in these stars such as diffusion, gravitational settling and radiative levitation.

We have organized the research material in this special issue around five main themes, hot subdwarfs as stellar populations, their origin, their composition, their pulsational characteristics and their evolution within binary systems and the nature of their companions.

Origins: The origin of hot subdwarfs was a mystery until many were identified to be members of binary systems. The problem is that there is no accepted mechanism for a single star to lose most of its hydrogen envelope before core-helium ignition. Binarity provides a number of natural mechanisms for achieving this. If the initially more massive (primary) member of a binary pair fills its Roche lobe near the tip of the first giant branch (i.e. shortly before helium ignition), its hydrogen envelope will be stripped away. This may be due either to stable Roche lobe overflow onto the companion, or to the ejection of a common envelope which forms around both stars; both leave a nearly-naked helium core. Following helium ignition, a hot subdwarf is formed with a binary companion. Binary star evolution may also produce helium white-dwarf pairs; if such a pair merges following orbital decay, a single hot subdwarf may be formed. These scenarios are now relatively well established, but puzzles still exist. Why is the binary fraction of EHB stars low in globular clusters? Why are single EHB stars not fast rotators? Some subdwarfs appear to have substellar companions, i.e. a brown dwarf or a planet. Do such companions play a rôle in the formation of the hot subdwarf? Can a single red giant have an enhanced stellar wind strong enough to produce a hot subdwarf?

Hot subdwarfs as stellar populations: Hot subdwarfs comprise the dominant population in surveys of blue objects 
and are thus important for our understanding of the structure and evolution of the Galaxy. They are found in the thin disk, the thick disk, the halo and the bulge, and are therefore members of all Galactic populations. One hot subdwarf, US 708, has been discovered among dozens of hyper-velocity stars, stars which move so fast that they are unbound to the Galaxy. Other hot subdwarf hyper-velocity candidates are being discovered; their origin is a dilemma. As hot subdwarfs provide an important source of far-ultraviolet (FUV) light in the Galaxy, it is expected that they also contribute significantly to the FUV in elliptical galaxies, which are believed to evolve passively and only to contain old stars. Hot subdwarfs are indeed a probe in the study of elliptical galaxies.

Hot subdwarfs in binary systems: Hot subdwarfs in binary systems provide insight into stellar astrophysics as both components are coeval and their physical parameters can be determined. The companions of hot subdwarfs include planets, brown dwarfs, main sequence stars, white dwarfs, and, possibly, neutron stars or black holes. Even more remarkably, HW Vir, an eclipsing binary, appears to have two circumbinary planets. Such stars help us to explore fundamental processes in the evolution of many binary systems, including common-envelope evolution, magnetic braking, and so on. Ultimately, a hot subdwarf with a sufficiently massive white dwarf companion may evolve to explode as a type Ia supernova (SN Ia) explosion-one of many possible origins for these conspicuous cosmological-distance indicators.

Chemical compositions: The chemical composition of the atmospheres of hot subdwarfs is still puzzling. In most sdB stars, light elements (with $Z<20$ ) are depleted while the remainder are enriched (iron excepted). Some sdB stars and many sdO stars are strongly enriched in helium, nitrogen and sometimes carbon, and show other chemical peculiarities. Spectral analysis is made with high-precision spectra and state-of-art model atmospheres, in which departures from local thermodynamic equilibrium (LTE) and line blanketing are taken into account. In addition, the study of composition anomalies requires a proper understanding of other physical processes operating from the upper atmosphere to the deep interior, including diffusion, gravitational settling, radiative levitation, radiatively-accelerated winds, convective mixing, and the consequences of pulsations. Extreme composition peculiarities may provide pointers to the previous history, or formation channel, for certain classes of hot subdwarf.

Astroseismology of hot subdwarfs: Asteroseismology is an excellent tool to probe the interiors of stars; a few dozens of hot subdwarfs have been found to be multimode pulsators. There are several families; sdB stars include pressure $(p)$-mode, gravity $(g)$-mode, and hybrid pulsators, whilst two sdO stars also pulsate. $p$-mode pulsators are characterized by low-amplitude, multi-periodic, shortperiod (80-600s) light variations, $g$-mode pulsators show low-amplitude, multi-periodic, long-period (2000 to $9000 \mathrm{~s}$ ) light variations, while hybrid pulsators display both $p$ - and $g$-mode characteristics. The significance of asteroseismology is that it enables a star's mass to be measured directly, whether the star is a member of a binary or not. The mass distribution of $\mathrm{sdB}$ pulsators measured in this way can then be compared with the distributions of masses predicted by stellar evolution theory.

Over the last decade, progress in all these areas has been very rapid. We hope that the synopsis of recent research presented in this Special Issue of Astrophysics \& Space Science is timely, and we trust it will prove of lasting value to the community. 